\title{
Originals
}

\section{Cardiac Autonomic Neuropathy in Diabetes: Comparison of Measures of R-R Interval Variation}

\author{
D. J. Ewing, D. Q. Borsey, F. Bellavere* and B. F. Clarke \\ University Department of Medicine and Diabetic and Dietetic Department, Royal Infirmary, Edinburgh, UK
}

Summary. Five different methods of analysing R-R interval (heart rate) variation were compared, using a computer technique, in 61 diabetics with a wide range of responses to autonomic function testing. Two methods differentiated best between the diabetics with and without autonomic damage: (1) the standard deviation of the mean R-R interval recorded for $5 \mathrm{~min}$ during quiet breathing with the subject either sitting or standing; (2) the difference between the maximum and minimum heart rates recorded over $1 \mathrm{~min}$ during deep breathing at six breaths per minute, again with the subject either sitting or standing. For routine clinical usage we conclude that recording the heart rate for $1 \mathrm{~min}$ on an ECG, while the subject sits and breaths deeply at six breaths per minute, and then measuring the difference between the maximum and minimum heart rate, is the most practical method currently available. For research purposes either this method or the standard deviation method during quiet breathing for $5 \mathrm{~min}$, should be used.

Key words: Diabetes mellitus, autonomic neuropathy, R-R interval variation, tests of autonomic function

Beat-to-beat ( $R-R$ interval) variation in heart rate has been recognised for many years, but it was only in 1973 that its use as a measure of the integrity of the autonomic nervous system was first described [1]. Wheeler and Watkins [1] showed that beat-to-beat heart rate variation during deep breathing, measured with a heart rate monitor, was diminished in some diabetics and attributed this to vagal neuropathy since atropine, but not propranolol, abolished the

\footnotetext{
* Present address: Instituto di Medicina Clinica, Cattedra di Gerontologia e Malattie del Ricambio Policlinico, via Giustiniani, Padova, Italy
}

heart rate variation in normal subjects. Subsequently several different methods of recording and analysing beat-to-beat variation have been described. These have included the computer analysis of a 5-min period of resting heart rate during normal breathing, with calculation of the standard deviation of the mean heart rate over that period [2]; and measurement of the differences between successive R-R intervals obtained by an ECG with derivation of the 'mean square successive difference' [3]. Modifications of Wheeler and Watkins' original method of measuring beat-to-beat variation during $1 \mathrm{~min}$ of deep breathing have been described by Sundkvist et al. [4] and Hilsted and Jensen [5] using an ECG.

Although measurement of beat-to-beat heart rate variation is now generally accepted as a valid test of cardiac parasympathetic function $[6,7]$ there is still considerable debate as to which of the methods currently available is the most useful for general clinical use [8]. Doubts have also been expressed about the widespread and often non-critical acceptance of beat-to-beat variation as a test of autonomic function without adequate consideration of its reproducibility and usefulness, particularly in subjects with borderline results [9].

Accordingly, this study was designed to assess which of the methods currently available for measuring beat-to-beat heart rate variation is most suitable as a test of cardiac parasympathetic function in diabetics.

\section{Subjects}

Sixty-one diabetics, predominantly male ( 57 male, 4 female) were selected to obtain as wide a range of responses to autonomic function tests as possible. Their ages ranged from 25 to 69 years and the duration of diabetes from 2 to 39 years. Fifty-three subjects were on insulin and the remainder on oral hypoglycaemic agents. None of the subjects had symptoms or clinical signs of cardiac failure or respiratory disease and none was on treatment for hyper- 
tension. As part of another study each subject had a $24 \mathrm{~h} \mathrm{ECG}$ tape recording, and all subjects were in sinus rhythm throughout the $24 \mathrm{~h}$ period. There was no evidence of sinus arrest or alternating bradycardia and tachycardia suggestive of the "sick sinus syndrome' in any of the subjects.

Before beat-to-beat ( $R-R$ interval) heart rate was recorded, each subject performed four other autonomic function tests. These were the heart rate responses to the Valsalva manoeuvre and standing (assessing cardiac parasympathetic function); and the blood pressure responses to sustained handgrip and standing (assessing more widespread sympathetic damage). Details of these tests and their applicability in the assessment of autonomic function have been described elsewhere $[6,7,9]$.

On the basis of these tests, the subjects were divided into three groups:

1) those with normal autonomic function tests (group $1, n=22$ )

2) those with abnormalities of autonomic function tests suggestive of cardiac parasympathetic damage only (either or both the Valsalva ratio or the $30 / 15$ lying-standing heart rate ratio abnormal, or both tests borderline) (Abnormal PS, group 2, $n=14$ )

3) those with abnormalities of autonomic function tests suggestive of cardiac parasympathetic and more widespread sympathetic damage (both the Valsalva ratio and 30/15 ratio abnormal, and either or both the response to sustained hand grip and the postural fall in blood pressure abnormal, or both responses borderline) (Abnormal PS + S, group 3, $n=25$ ).

Table 1 gives the ages, duration of diabetes and mean responses to autonomic function tests in the three groups.

\section{Methods}

\section{$R-R$ Interval Recording Technique}

Each subject's ECG was recorded onto magnetic tape as described previously $[2,10]$. Three chest electrodes were used to minimize interference. A marker signal was used to indicate the start and finish of the study periods (see below) and recorded on a second channel of the tape recorder.

\section{Protocol}

Each subject had six study periods on each of two successive days, using a standard protocol. The ECG was recorded in three different positions - lying, sitting and standing, during both quiet and deep breathing. The order in which lying, sitting and standing were recorded was randomly allocated between the patients. In each position, after a period of $3 \mathrm{~min}$ quiet rest, the heart rate was recorded for a 5 -min sample while the patient breathed quietly, and after a further 2-min interval the heart rate was recorded during a 1-min sample of deep breathing at 6 breaths $/ \mathrm{min}$. On day 2 the protocol was repeated with the positions recorded in the same order. Before ECG recording began on day 1, each subject was given a training period of deep breathing at 6 breaths $/ \mathrm{min}$ ( $5 \mathrm{~s}$ inspiration and $5 \mathrm{~s}$ expiration).

Seven subjects were recorded on one day only; three were unable to return for a second visit, and in the other four the recordings on day 2 were technically faulty.

\section{Computer Analysis of $R-R$ Interval}

The magnetic tapes were replayed at $60 \times$ real speed and fed into a purpose built $R-R$ interval analyser for accurate timing of the $R-R$ intervals. A digital and punch-tape print-out of successive
R-R intervals was produced for each of the 12 study periods in all subjects. The punch tape was later fed into a PDP12 computer for further analysis. This computer was programmed to analyse each sample for number of heart beats, number of artefacts, mean $R-R$ interval of the sample, standard deviation of the mean R-R interval, mean square successive difference and mean successive difference of the $\mathrm{R}-\mathrm{R}$ intervals in the sample, mean heart rate, maximum and minimum $R-R$ interval and heart rate, ratio of maximum to minimum R-R interval and heart rate. Of the 690 study periods analysed, 14 ( $2 \%$ ) records (from 11 different subjects) had to be discarded as the samples were too short or had too many artefacts.

\section{$R-R$ Interval Variation Analysis}

The following measures of $R-R$ interval variation were made:

1) Standard deviation (SD) of the mean R-R interval of the sample period. This measure gives an indication of the spread around the mean value and hence an indication of the variability of the heart rate during a short sampling period, provided that the mean heart rate remains relatively constant.

2) Mean square successive difference (MSSD) of the R-R intervals recorded during the sample period. This is measured by calculating the mean of the squares of differences between successive $\mathrm{R}-\mathrm{R}$ intervals. It gives an indication of the inter-beat differences and is a measure of actual beat-to-beat variation.

3) Mean successive difference (MSD) of the R-R intervals recorded during the sample period. This measure is derived in the same way as method (2) except that successive differences are not squared, making it simpler to calculate.

4) Difference between the maximum and minimum heart rate (Max-Min HR) during the sample period. This measures the amplitude of the heart rate variation and is particularly applicable where a stimulus, such as deep breathing at 6 breaths $/ \mathrm{min}$, increases the heart rate variation in normal subjects but not in subjects with vagal damage.

5) Ratio of the longest to the shortest R-R interval during the sample period (Mas/Min R-R).

\section{Statistical Analysis}

The results obtained from each subject were later fed into a further computer programmed for statistical analysis of the data using a variety of standard techniques including analysis of variance, correlation coefficients, and Student's paired ' $t$ ' test. The mean values for the sample periods on the two successive days were used to calculate the analysis of variance and the correlation coefficients, except in those subjects where only one recording of a particular sample period was available, when the single value was used. The results are expressed as mean \pm SEM.

\section{Results}

\section{Reproducibility of the Different Methods to Measure $R-R$ Interval Variation}

The results of the two successive days were compared for the five different measures of $R-R$ interval variation. In all three positions, both during quiet and during deep breathing there were no statistically significant differences in 29 of the 30 results. The only 
Table 1. Ages, duration of diabetes and results of autonomic function tests in the three groups of diabetics (mean \pm SEM)

\begin{tabular}{|c|c|c|c|c|c|}
\hline & $\begin{array}{l}\text { Group } 1 \\
\text { (normal) } \\
(n=22)\end{array}$ & $\begin{array}{l}\text { Group } 2 \\
\text { (parasympathetic } \\
\text { neuropathy) } \\
(n=14)\end{array}$ & $\begin{array}{l}\text { Group } 3 \\
\text { (parasympathetic } \\
\text { and sympathetic } \\
\text { neuropathy) } \\
(n=25)\end{array}$ & $\begin{array}{l}\text { F } \\
\text { ratio }\end{array}$ & Significance \\
\hline $\begin{array}{l}\text { Age } \\
\text { (years) } \\
\text { (range) }\end{array}$ & $\begin{array}{l}43.9 \pm 2.6 \\
(25-69)\end{array}$ & $\begin{array}{l}46.4 \pm 3.2 \\
(25-66)\end{array}$ & $\begin{array}{l}45.8 \pm 2.4 \\
(27-67)\end{array}$ & 0.2 & NS \\
\hline $\begin{array}{l}\text { Duration of diabetes } \\
\text { (years) } \\
\text { (range) }\end{array}$ & $\begin{array}{l}12.7 \pm 1.9 \\
(2-31)\end{array}$ & $\begin{array}{l}20.6 \pm 2.4 \\
(10-28)\end{array}$ & $\begin{array}{l}18.8 \pm 1.8 \\
(1-39)\end{array}$ & 4.2 & $p<0.05$ \\
\hline Valsalva ratio & $1.59 \pm 0.05$ & $1.20 \pm 0.06$ & $1.05 \pm 0.04$ & 34.1 & $p<0.01$ \\
\hline $\begin{array}{l}\text { Lying to } \\
\text { standing } \\
30 / 15 \text { heart } \\
\text { rate ratio }\end{array}$ & $1.12 \pm 0.01$ & $1.00 \pm 0.02$ & $1.00 \pm 0.01$ & 10.4 & $p<0.01$ \\
\hline $\begin{array}{l}\text { Increase in } \\
\text { diastolic blood pressure } \\
\text { during handgrip } \\
(\mathrm{mmHg})\end{array}$ & $32 \pm 2.6$ & $31 \pm 3.3$ & $17 \pm 2.5$ & 20.2 & $p<0.01$ \\
\hline $\begin{array}{l}\text { Decrease in } \\
\text { systolic blood pressure } \\
\text { on standing } \\
(\mathrm{mmHg})\end{array}$ & $4 \pm 3.1$ & $9 \pm 3.8$ & $26 \pm 2.9$ & 15.4 & $p<0.01$ \\
\hline
\end{tabular}

NS $=$ Not significant

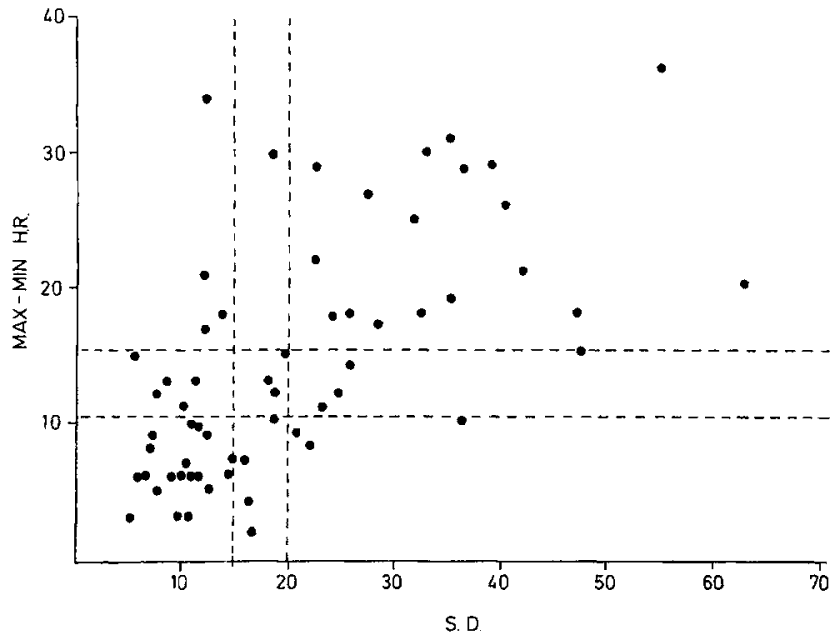

Fig. 1. Comparison between maximum-minimum heart rate (MaxMin HR) (beats/min) (lying deep breathing) [1] and standard deviation (SD) of mean R-R interval (ms) (standing quiet breathing) [2] in 61 diabetics. The areas between the dotted lines represent the 'borderline' zones for each test $(r=0.62)$

exception was during quiet breathing lying with MaxMin HR $(p<0.05)$. The measurements of successive differences of R-R interval (MSSD and MSD) correlated extremely closely $(r=0.99)$. In view of this, only the MSSD results are included in the tables. Similarly, the two measures of maximum and minimum heart rate, the Max-Min HR and the Max/Min R-R also correlated closely $(r=0.97)$. Consequently only Max-Min HR is included subsequently as this is a more widely accepted measure of $\mathbf{R}-\mathbf{R}$ interval variation. The measures are therefore reproducible if repeated on successive days.

\section{Results of $R-R$ Interval Variation in the Three Subject Groups}

The mean results for SD, MSSD and Max-Min HR for the three groups of subjects are included in Table 2. As can be seen, analysis of variance showed good separation between the groups with both SD and Max-Min HR but MSSD showed mainly nonsignificant results. The ' $F$ ' ratio reflects overall differences between groups and identifies variation whatever the source. In this case, the main source of the variation was between group I (normal) and the other two groups, both of whom had parasympathetic damage. The highest $F$ ratios for SD were sitting and standing while breathing quietly; and for Max-Min HR sitting both breathing quietly and breathing deeply, and standing breathing deeply (Table 2). 
Table 2. Results of different measures of $R-R$ interval variation in the three groups of diabetics (mean \pm SEM)

\begin{tabular}{|c|c|c|c|c|c|}
\hline & $\begin{array}{l}\text { Group } 1 \\
\text { (normal) } \\
(n=22)\end{array}$ & $\begin{array}{l}\text { Group } 2 \\
\text { (parasympathetic } \\
\text { neuropathy) } \\
(n=14)\end{array}$ & $\begin{array}{l}\text { Group } 3 \\
\text { (parasympathetic } \\
\text { and sympathetic } \\
\text { neuropathy) } \\
(n=25)\end{array}$ & $\begin{array}{l}F \\
\text { ratio }\end{array}$ & Significance \\
\hline \multicolumn{6}{|l|}{$\begin{array}{l}\text { Quiet rest } \\
\text { for } 5 \mathrm{~min}\end{array}$} \\
\hline $\begin{array}{l}\text { Lying } \\
\text { SD (ms) } \\
\text { MSSD (ms) } \\
\text { Max-Min HR (beats/min) }\end{array}$ & $\begin{array}{l}33.4 \pm 2.5 \\
16.9 \pm 2.6 \\
20.4 \pm 1.5\end{array}$ & $\begin{array}{r}18.2 \pm 3.1 \\
9.6 \pm 3.3 \\
13.5 \pm 1.8\end{array}$ & $\begin{array}{l}19.1 \pm 2.3 \\
14.2 \pm 2.5 \\
12.5 \pm 1.4\end{array}$ & $\begin{array}{r}11.1 \\
1.4 \\
8.3\end{array}$ & $\begin{array}{l}p<0.01 \\
\text { NS } \\
p<0.01\end{array}$ \\
\hline $\begin{array}{l}\text { Sitting } \\
\text { SD } \\
\text { MSSD } \\
\text { Max-Min HR }\end{array}$ & $\begin{array}{l}33.3 \pm 2.4 \\
14.7 \pm 1.6 \\
22.8 \pm 1.5\end{array}$ & $\begin{array}{r}16.0 \pm 3.0 \\
8.7 \pm 2.1 \\
14.1 \pm 1.9\end{array}$ & $\begin{array}{l}17.6 \pm 2.2 \\
11.7 \pm 1.5 \\
12.8 \pm 1.4\end{array}$ & $\begin{array}{r}15.3 \\
2.7 \\
12.4\end{array}$ & $\begin{array}{l}p<0.01 \\
\text { NS } \\
p<0.01\end{array}$ \\
\hline $\begin{array}{l}\text { Standing } \\
\text { SD } \\
\text { MSSD } \\
\text { Max-Min HR }\end{array}$ & $\begin{array}{l}30.2 \pm 2.1 \\
12.9 \pm 1.4 \\
24.0 \pm 1.7\end{array}$ & $\begin{array}{r}14.3 \pm 2.6 \\
7.8 \pm 1.7 \\
15.0 \pm 2.2\end{array}$ & $\begin{array}{l}16.0 \pm 1.9 \\
10.6 \pm 1.3 \\
14.9 \pm 1.6\end{array}$ & $\begin{array}{r}16.3 \\
2.6 \\
8.8\end{array}$ & $\begin{array}{l}p<0.01 \\
\text { NS } \\
p<0.01\end{array}$ \\
\hline $\begin{array}{l}\text { Deep breathing } \\
\text { for } 1 \mathrm{~min}\end{array}$ & & & & & \\
\hline $\begin{array}{l}\text { Lying } \\
\text { SD } \\
\text { MSSD } \\
\text { Max-Min HR }\end{array}$ & $\begin{array}{l}44.4 \pm 4.2 \\
29.4 \pm 4.3 \\
19.0 \pm 1.7\end{array}$ & $\begin{array}{l}24.5 \pm 5.3 \\
14.6 \pm 5.4 \\
13.5 \pm 2.1\end{array}$ & $\begin{array}{l}21.0 \pm 4.0 \\
18.2 \pm 4.1 \\
11.0 \pm 1.6\end{array}$ & $\begin{array}{l}8.3 \\
2.8 \\
6.2\end{array}$ & $\begin{array}{l}p<0.01 \\
\text { NS } \\
p<0.01\end{array}$ \\
\hline $\begin{array}{l}\text { Sitting } \\
\text { SD } \\
\text { MSSD } \\
\text { Max-Min HR }\end{array}$ & $\begin{array}{l}35.4 \pm 3.0 \\
19.7 \pm 2.2 \\
18.8 \pm 1.3\end{array}$ & $\begin{array}{l}21.7 \pm 3.8 \\
11.1 \pm 2.7 \\
12.6 \pm 1.7\end{array}$ & $\begin{array}{r}16.0 \pm 2.8 \\
12.0 \pm 2.1 \\
9.0 \pm 1.2\end{array}$ & $\begin{array}{r}11.4 \\
4.0 \\
12.1\end{array}$ & $\begin{array}{l}p<0.01 \\
p<0.05 \\
p<0.01\end{array}$ \\
\hline $\begin{array}{l}\text { Standing } \\
\text { SD } \\
\text { MSSD } \\
\text { Max-Min HR }\end{array}$ & $\begin{array}{l}31.2 \pm 2.8 \\
15.6 \pm 1.9 \\
21.7 \pm 1.6\end{array}$ & $\begin{array}{l}20.0 \pm 3.5 \\
10.1 \pm 2.4 \\
14.3 \pm 2.0\end{array}$ & $\begin{array}{l}15.3 \pm 2.6 \\
10.4 \pm 1.8 \\
11.3 \pm 1.5\end{array}$ & $\begin{array}{r}9.1 \\
2.1 \\
11.1\end{array}$ & $\begin{array}{l}p<0.01 \\
\text { NS } \\
p<0.01\end{array}$ \\
\hline
\end{tabular}

$\mathrm{NS}=$ not significant; $\mathrm{SD}=$ standard deviation of mean $\mathrm{R}-\mathrm{R}$ interval (ms); MSSD = mean square successive differences of $\mathrm{R}-\mathrm{R}$ interval (ms); Max-Min $\mathrm{HR}=$ difference between maximum and minimum heart rates (beats $/ \mathrm{min}$ )

\section{Correlation Between the Different Measures of $R-R$ Interval Variation and Other Tests of Autonomic Function}

Table 3 gives the correlation coefficients between the different measures of $R-R$ interval variation and the other tests of autonomic function in the whole group of 61 diabetics. As can be seen SD correlated well with the Valsalva ratio and the lying to standing $30 / 15$ heart rate ratio (both measures of cardiac parasympathetic function) in all three positions both during quiet and during deep breathing, as did MaxMin HR. These measures also correlated, although not as closely, with the blood pressure response to sustained handgrip and the postural fall in blood pressure (both indicating wider sympathetic damage). As with the analysis of variance, MSSD showed poor results and only correlated with the $30 / 15$ ratio.
There was a slight, but significant correlation in some positions between age and both SD and Max-Min $\mathrm{HR}$; while duration of diabetes correlated slightly more closely with both measures.

\section{Mean Heart Rate}

Table 3 also lists the correlation between mean heart rate in each position and the various indices of heart rate variation. As can be seen from the table SD and MSSD correlated with the mean heart rate, unlike Max-Min HR.

\section{Relationship Between SD and Max-Min HR}

SD has previously been measured in the standing position while breathing quietly [2] and Max-Min HR in the lying position while breathing deeply [1]. 
Table 3. Relationship between different measures of R-R interval variation and other tests of autonomic function in 61 diabetics (correlation coefficient $r$ given in Table)

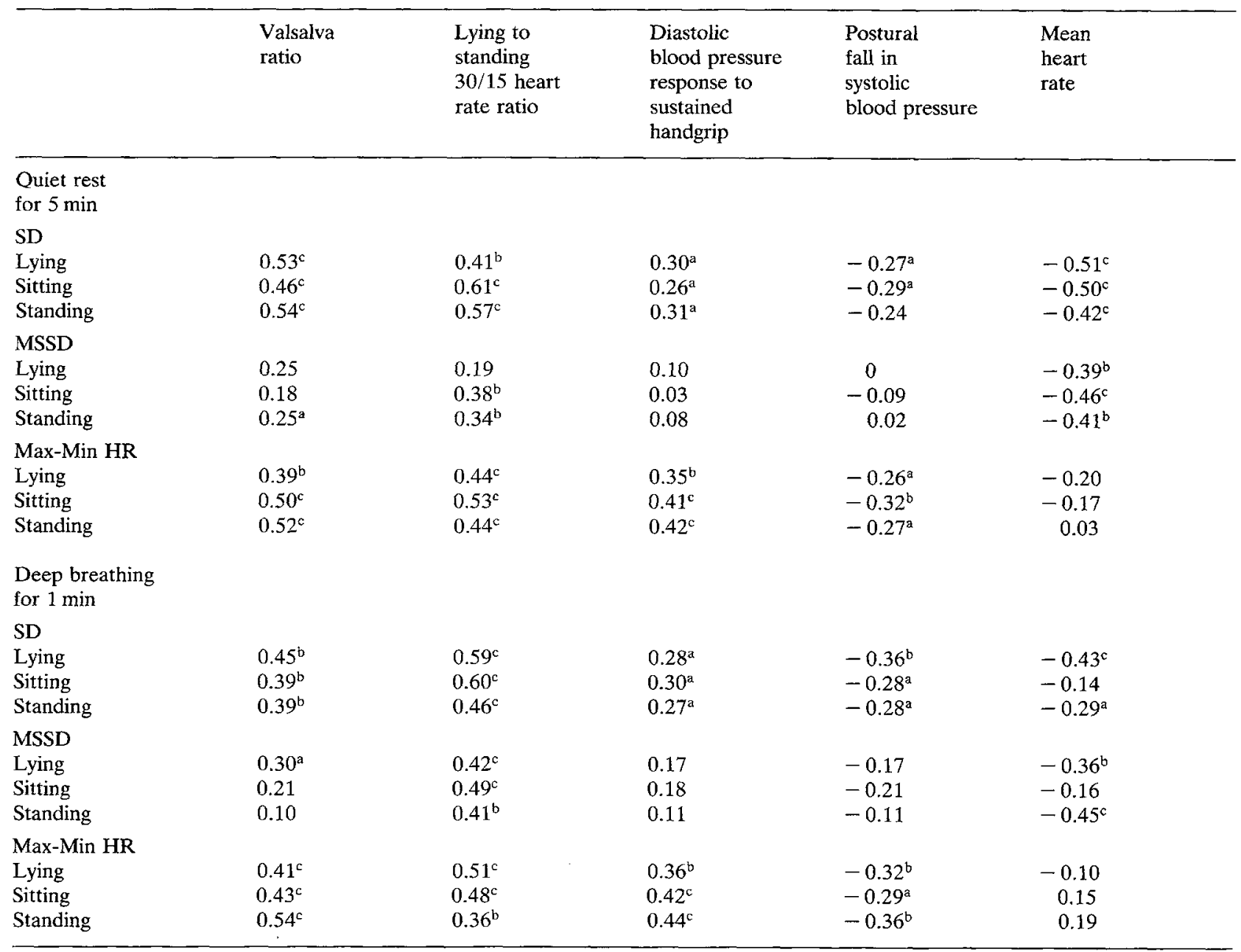

$\mathrm{a}=p<0.05 ; \mathrm{b}=p<0.01 ; \mathrm{c}=p<0.001 ; \mathrm{SD}=$ standard deviation of mean $\mathrm{R}-\mathrm{R}$ interval (ms); MSSD = mean square successive differences of $\mathrm{R}-\mathrm{R}$ interval (ms); Max-Min $\mathrm{HR}=$ difference between maximum and minimum heart rates (beats $/ \mathrm{min}$ ).

In view of this, the two measures were compared in these positions in the 61 subjects, and Fig. 1 shows the individual results from the first study day. There was a close correlation between the two measures $(r$ $=0.62$ ). Of the individual results, 19 were abnormal with both measures, and 18 normal with both measures. In only seven subjects was there a discrepancy - four with normal Max-Min HR and abnormal SD and three with abnormal Max-Min HR and normal SD. The remaining results were in the previously defined borderline zones of one or other test.

\section{Discussion}

This present analysis of different measures of R-R interval variation is the first comprehensive comparison of the currently available tests. Opportunity was taken to compare the lying, sitting and standing positions both while breathing quietly and while breathing deeply. As can be seen from the results, all measures were reproducible on the two successive days.

The five measures of $R-R$ interval variation are based on three different ways of assessing and calculating the variation in heart rate over a short period. The first, which is measurement of the SD of the mean heart rate, has been used by us previously in diabetics [2] and by others in assessment of heart rate variation in other situations [11]; and provides a numerical estimate of the spread about the mean heart rate. It is, however, only applicable if the mean heart rate is relatively constant. If heart rate changes markedly during recording, the SD will give erroneous results, and therefore it is more reliable over relatively short periods. For this reason we have pre- 
viously estimated heart rate variation by this method for approximately $5 \mathrm{~min}$, with a rest period before recording to allow the heart rate to settle to a steady level [2].

Measurement of inter-beat differences can be assessed by the method first described by Gundersen and Neubauer [3] for the MSSD. This measure will detect changes in the successive beat differences, and is independent of the trends in heart rate. As such, therefore, it estimates true "beat-to-beat" variation. MSD has not previously been measured, but is simpler to calculate than MSSD. However as can be seen the results were almost identical to MSSD.

The third way of assessing $R-R$ interval variation is during deep breathing at 6 breaths/min with measurement of the maximum-minimum heart rate. This particular rate of breathing is used as it has been shown that in normal subjects this produces the maximum variation in heart rate $[12,13]$ and increases the separation between normal and abnormal in diabetics. The difference between maximum and minimum heart rate during deep breathing is now widely used as a test for autonomic neuropathy since its initial description by Wheeler and Watkins [1]. Sundkvist et al. [4] have produced a simple formula ( $\left.{ }^{\operatorname{ax}} /{ }_{\text {Min }} R-R\right)$ for calculating this from an ECG, but as can be seen from our results this correlates almost exactly with the more widely used maximumminimum HR. Hilsted and Jensen [5] have shown that ECG measurements of maximum and minimum heart rates during deep breathing are reliable and reproducible.

Examination of our results in the three groups of diabetics shows that MSSD did not give good separation between normal and abnormal in contrast to SD and Max-Min HR. Sitting and standing during quiet breathing for SD, and during deep breathing for Max-Min HR gave the best results. When compared with other recognised tests of cardiovascular reflex function MSSD again did not correlate closely. There is of course no absolute standard against which the various methods of assessing $R-R$ interval variation can be judged but we have taken the responses to the Valsalva manoeuvre and sustained handgrip, the lying to standing 30/15 heart ratio and the postural fall in blood pressure as standards here. It was to be expected that there would be a closer correlation with tests of cardiac vagal function (Valsalva manoeuvre and $30 / 15$ ratio) than with tests of more widespread sympathetic involvement (handgrip and postural hypotension) and this is, in fact, the case.

One other feature to come out of our analysis is that whereas the measures using $\mathrm{R}-\mathrm{R}$ interval (SD and MSSD) show a relationship with the mean heart rate, Max-Min HR is independent of the resting heart rate. This means that irrespective of the resting heart rate, Max-Min HR can be used to discern normal from abnormal whereas the resting heart rate has to be considered in calculations involving SD.

From the results of this study we would conclude that $R-R$ interval variation as a test of cardiac parasympathetic function is best measured by the MaxMin HR during deep breathing in either the sitting or standing position, or by the SD during quiet breathing in the same positions. In routine clinical practice we would now advocate that Max-Min HR using an ECG as described by Hilsted and Jensen [5] is probably the most practical method of assessing $R-R$ interval variation that is currently available and this should be performed sitting as this is most convenient for the patient. Normal ranges have previously been defined with abnormal as less than 10 beats/min variation $[13,14]$ although these were taken in the lying position. For research purposes however, we would advocate either this method or SD during quiet breathing while standing, where normal ranges have also been clearly defined $[2,15]$.

Despite our recommendations about heart rate variation there are some pitfalls for the unwary. Reproducibility in normal subjects using SD has been shown to be poor [7] and interpretation of borderline results of $R-R$ interval variation is difficult as illustrated by our results. If the results are abnormal the interpretation is easy as both SD or Max-Min HR give consistent results. If however, the results are borderline, interpretation is more difficult. In these circumstances and indeed in all circumstances where autonomic function is assessed, we would stress the importance of doing a battery of autonomic function tests that reflect both parasympathetic and sympathetic damage and not just relying on any one test. In our current practice we use the Valsalva manoeuvre, the lying to standing $30 / 15$ heart rate ratio, sustained handgrip, and the postural fall in blood pressure as well as one measure of $\mathrm{R}-\mathrm{R}$ interval variation. We are now adopting the maximum-minimum heart rate recorded during deep breathing at 6 breaths/min, and measured from an ECG, in our standard clinical assessment of autonomic function.

Acknowledgements. We thank Dr. J. M. M. Neilson, Department of Medical Physics, University of Edinburgh, for his help in measuring and analysing the R-R interval results; and Dr. R. Prescott and Mr. W. Adams, Medical Computing and Statistics Unit, University of Edinburgh, for their help in the statistical analysis. Dr. D. J. Ewing holds a Wellcome Trust Senior Lectureship.

\section{References}

1. Wheeler $\mathbf{T}$, Watkins PJ (1973) Cardiac denervation in diabetes. Br Med J 4: 584-586

2. Murray A, Ewing DJ, Campbell IW, Neilson JMM, Clarke BF 
(1975) R-R interval variations in young male diabetics. $\mathrm{Br}$ Heart J 37: 882-885

3. Gundersen HJG, Neubauer B (1977) A long term autonomic nervous abnormality. Reduced variations in resting heart rate measured by a simple and sensitive method. Diabetologia 13: $137-140$

4. Sundkvist G, Almer L-O, Lilja B (1979) Respiratory influence on heart rate in diabetes mellitus. Br Med J 1: 924-925

5. Hilsted J, Jensen SB (1979) A simple test for autonomic neuropathy in juvenile diabetics. Acta Med Scand 205: 385-387

6. Clarke BF, Ewing DJ, Campbell IW (1979) Diabetic autonomic neuropathy. Diabetologia 17: 195-212

7. Ewing DJ (1978) Cardiovascular reflexes and autonomic neuropathy. Clin Sci Mol Med 55: 321-327

8. Bennett T, Farquhar IK, Hosking DJ, Hampton JR (1978) Assessment of methods for estimating autonomic nervous control of the heart in patients with diabetes mellitus. Diabetes 27: $1167-1174$

9. Ewing DJ, Campbell IW, Clarke BF (1980) Assessment of cardio vascular effects in diabetic autonomic neuropathy and prognostic implications. Annals Intern Med 92: 308-311

10. Ewing DJ, Campbell IW, Murray A, Neilson JMM, Clarke BF (1978) Immediate heart rate response to standing: a simple test for autonomic neuropathy in diabetes. $\mathrm{Br}$ Med $\mathrm{J} 1$ : 145-147
11. Cashman PMM (1977) The use of R-R interval and difference histograms in classifying disorders of sinus rhythm. J Med Eng Technol 1: 20-28

12. Angelone A, Coulter NA (1964) Respiratory sinus arrhythmias: a frequency dependent phenomenon. J Appl Physiol 19: 479-482

13. Watkins PJ, Mackay JD (1980) Cardiac denervation in diabetic neuropathy. Annals Intern Med 92: 304-307

14. Page MMcB, Watkins PJ (1977) The heart in diabetes: autonomic neuropathy and cardiomyopathy, Clin Endocrinol Metab 6: 377-388

15. Campbell IW, Ewing DJ, Clarke BF (1979) Tests of cardiovascular reflex function in diabetic autonomic neuropathy. In: Gries FA, Freund HJ, Rube F, Berger $H$ (eds) Aspects of autonomic neuropathy in diabetes. Georg Thieme Verlag, Stuttgart, pp 61-68

Received: 11 November 1980

and in revised form: 5 February 1981

Dr. D. J. Ewing

University Department of Medicine

Royal Infirmary,

Edinburgh EH3 9YW, UK 